\title{
Molecular characterization of testicular germ cell tumors: chasing the underlying pathways
}

\author{
Michal Chovanec ${ }^{*, 1,2} \&$ Liang Cheng $* *, 3,4$ \\ ${ }^{1}$ Second Department of Oncology, Faculty of Medicine, Comenius University \& National Cancer Institute, Klenova 1, 83310 \\ Bratislava, Slovakia \\ ${ }^{2}$ Division of Hematology Oncology, Indiana University Simon Cancer Center, Indianapolis, 535 Barnhill drive, Indiana University \\ School of Medicine, Indianapolis, IN 46202, USA \\ ${ }^{3}$ Departments of Pathology \& Laboratory Medicine, Indiana University School of Medicine, Indianapolis, IN 46202, USA \\ ${ }^{4}$ Department of Urology, Indiana University School of Medicine, Indianapolis, IN 46202, USA \\ *Author for correspondence: michal.chovanec1@gmail.com; Twitter: @mikechovanec \\ **Author for correspondence: liang_cheng@yahoo.com
}

\section{"Finding predictive biomarkers leading to effective treatments is of great importance in refractory TGCT"'}

First draft submitted: 13 August 2018; Accepted for publication: 12 September 2018; Published online: 7 December 2018

Keywords: biomarkers $\bullet$ molecular genetics $\bullet$ TCGA $\bullet$ testicular germ cell tumors $\bullet$ testis $\bullet$ The Cancer Genome Atlas

Testicular germ cell tumors (TGCT) are the most common cancer in the population of young men [1]. Pioneering efforts of researchers and clinicians from the 1960s to the 1980s have led to a cure rate of about $80 \%$ for patients with metastatic disease [2]. This success is attributed to the unique sensitivity to cisplatin and several other cytostatic agents such as bleomycin, etoposide, ifosfamide and paclitaxel [1]. However, the reasons for such exceptional outcomes are largely unknown.

The search for underlying mechanisms of TGCT pathogenesis is complicated by the histological variability of these tumors [3]. The biology of TGCT seems to differ from other solid malignancies. This difference stems from the developmental origins of TGCT. Primordial germ cells carrying the heritable genetic information stand in the center of TGCT pathogenesis. During the development of TGCT, they undergo a malignant transformation into a precursor lesion, a germ cell neoplasia in situ [4].

TGCT normally show aneuploid karyotypes, and a gain of the short arm of chromosome 12 has been identified as a cytogenetic hallmark biomarker [5,6]. Unlike most solid cancers, TGCT lack the high mutation rate, and the only driver mutations of KIT and KRAS were reported in a small proportion of patients, especially with seminoma [7-9]. The scientific evidence to support a role of a single-driver gene alteration in the pathogenesis of TGCT is insufficient. Rather, the polygenic nature of this disease is suggested where a certain gene expression profile may be associated with an increased risk of TGCT [10-12]. Furthermore, the pathology of TGCT is also associated with substantial epigenetic changes. Epigenetic signaling is one of the critical mechanisms in their development [13]; however, the exact driving moment of the switch to a malignant phenotype of primordial germ cells is unknown.

A recent The Cancer Genome Atlas (TCGA) study offers insight into the genomic landscape of TGCT [14]. The investigators provided comprehensive molecular characterization of 137 primary TGCT using high-dimensional genomic, epigenomic, transcriptomic and proteomic assays. They performed an initial histological evaluation classifying the tumor samples into seminomas and nonseminomas. Nonseminomas consisted of embryonal carcinomas, choriocarcinomas, yolk sac tumors, mature teratomas and immature teratomas [14]. Seminomas have shown a clear distinction from nonseminomas in the DNA methylation, mRNA, miRNA and proteomic profiling. The overall median frequency of mutations was $0.5 / \mathrm{Mb}$, which is in contrast to overall mutation rate of other cancers such as melanoma (14.4/Mb) [15], colorectal cancer (9.9-11.6/Mb) [16] or lung cancer (7.2/Mb) [17].

High-mutational burden is a well-established predictive biomarker for checkpoint inhibition in several cancers [18]; however, the scarcity of mutations in testicular cancer suggests a low probability to achieve a treatment effect with 
such agents. The present study showed a significant mutation rate in only three genes: KIT (18\%), KRAS (14\%) and NRAS (4\%), almost all exclusive to seminoma. Mutated KRAS was present in one patient with nonseminoma with $30 \%$ of seminomatous component. Current findings are consistent with previously reported data [9,19]. KITmutated seminomas have shown a phenotype with a strong immune infiltration that most likely contributes to an excellent prognosis in most of these tumors.

While PD-L1 status in tumor and immune cells is prognostic in TGCT [20,21], the authors did not discover a significant neoantigen signal. This fact and low-mutational burden may explain the lack of activity of the checkpoint inhibitor, pembrolizumab, in a Phase II study in refractory TGCT [22].

KIT gene, which is a potent driver of stem cell properties, and KIT protein were also highly expressed in seminomas. KIT gene expression was higher in KIT-mutated tumors. The low rate of mutations in seminoma do not provide an evidence of a universal driver for all TGCT. However, the most patients exhibiting these mutations had a history of undescended testicle. While cryptorchidism is a known risk factor, these results suggest that delayed testicular development could be associated with an increased risk of KIT, KRAS and NRAS mutations.

Dramatic differences were observed in global DNA methylation patterns within the histological subtypes [14]. Seminoma showed a completely unmethylated CpG DNA pattern. Embryonal carcinoma, on the other hand, showed extensive methylation at non- $\mathrm{CpG}(\mathrm{CpH})$ sites. $\mathrm{CpH}$ is suggested here as a potential diagnostic marker of embryonal carcinoma. DNA methylation patterns in nonseminomatous components were similar to the normal tissue development. The methylation patterns in nonseminoma were consistent with silencing of important tumorsuppressor genes such as BRCA1, RAD 51C, MGMT and RASSF1A (all involved in DNA repair pathways). Further epigenetic evaluation showed a high expression of miR-371a-3p in seminoma, and nonseminoma, but not in teratoma. This high-sensitivity biomarker is a novel predictor of the presence of TGCT as reported recently [23,24]. In addition, the authors reported a high expression of miR375 in teratoma and yolk sac tumor, but not in seminoma and embryonal carcinoma. miR375 may be the marker of chemotherapy resistance that is always seen in teratomas and frequently seen in the late relapse with yolk sac tumors. The identification of teratoma in the postchemotherapy residual tumor mass is one of the important clinical dilemmas. The potential utilization of plasmatic miR375 in this setting is yet to be determined.

The modern era of targeted treatments seems to evade TGCT. Due to their unique biological features and diversity, we were unable to establish novel treatments in TGCT that are also effective in other solid cancers. This paper provides a very important and extremely comprehensive overview of molecular characteristics of TGCT [14]. A number of seminomas seem to be influenced by KIT pathway alterations, predominantly with the KIT-PI3K enrichment. A polygenic nature of this disease is suggested with a strong importance of epigenetic signaling. An interesting premise for new treatment seems to be a demethylation of hypermethylated platinum-resistant tumors. One of the promising novel agents is the demethylating drug, guadecitabine. Guadecitabine was reported to overcome a cisplatin resistance in vitro [25], and the clinical Phase I trial is currently underway at Indiana University (NCT02429466). Finding predictive biomarkers leading to effective treatments is of great importance in refractory TGCT [26], as this entity is long without efficient alternatives. There is still a substantial 'unknown' in the biology of TGCT that calls for further intensive molecular research.

\section{Financial \& competing interests disclosure}

This work was supported by the Slovak Research and Development Agency under contract No. APVV-15-0086 and Scientific Grant Agency under contract number VEGA 1/0043/18 for M Chovanec. The authors have no other relevant affiliations or financialinvolvement with any organization or entity with a financial interest in or financial conflict with the subject matter or materials discussed in the manuscript apart from those disclosed.

No writing assistance was utilized in the production of this manuscript.

\section{References}

1. Cheng L, Albers P, Berney DM et al. Testicular cancer. Nat. Revs. Dis. Primers 4(1), 29 (2018).

2. Einhorn LH. Treatment of testicular cancer: a new and improved model. J. Clin. Oncol. 8(11), 1777-1181 (1990).

3. Cheng L, Lyu B, Roth LM. Perspectives on testicular germ cell neoplasms. Hum. Pathol. 59, 10-25 (2017).

4. Emerson RE, Cheng L. Premalignancy of the testis and paratestis. Pathology 45(3), 264-272 (2013).

5. Cheng L, Zhang S, Eble JN et al. Molecular genetic evidence supporting the neoplastic nature of fibrous stroma in testicular teratoma. Mod. Pathol. 25(10), 1432-1438 (2012). 
6. Cheng L, Zhang S, MacLennan GT et al. Interphase fluorescence in situ hybridization analysis of chromosome 12p abnormalities is useful for distinguishing epidermoid cysts of the testis from pure mature teratoma. Clin. Cancer Res. 12(19), 5668-5672 (2006).

7. McIntyre A, Summersgill B, Grygalewicz B et al. Amplification and overexpression of the KIT gene is associated with progression in the seminoma subtype of testicular germ cell tumors of adolescents and adults. Cancer Res. 65(18), 8085-8089 (2005).

8. Kemmer K, Corless CL, Fletcher JA et al. KIT mutations are common in testicular seminomas. Am. J. Pathol. 164(1), 305-313 (2004).

9. Litchfield K, Summersgill B, Yost $\mathrm{S}$ et al. Whole-exome sequencing reveals the mutational spectrum of testicular germ cell tumours. Nat. Commun. 6, 5973 (2015).

10. Brabrand $\mathrm{S}$, Johannessen $\mathrm{B}$, Axcrona $\mathrm{U}$ et al. Exome sequencing of bilateral testicular germ cell tumors suggests independent development lineages. Neoplasia 17(2), 167-174 (2015).

11. Litchfield $\mathrm{K}$, Loveday $\mathrm{C}$, Levy $\mathrm{M}$ et al. Large-scale sequencing of testicular germ cell tumour (TGCT) cases excludes major TGCT predisposition gene. Eur. Urol. 73(6), 828-831 (2018).

12. Rajpert-De Meyts E, Skotheim RI. Complex polygenic nature of testicular germ cell cancer suggests multifactorial aetiology. Eur. Urol. 73(6), 832-843 (2018).

13. Killian JK, Dorssers LC, Trabert B et al. Imprints and DPPA3 are bypassed during pluripotency- and differentiation-coupled methylation reprogramming in testicular germ cell tumors. Genome Res. 26(11), 1490-1504 (2016).

14. Shen H, Shih J, Hollern DP et al. Integrated molecular characterization of testicular germ cell tumors. Cell Rep. 23(11), 3392-3406 (2018).

15. Hodis E, Watson IR, Kryukov GV et al. A landscape of driver mutations in melanoma. Cell 150(2), 251-263 (2012).

16. Salem ME, Puccini A, Grothey A et al. Landscape of tumor mutation load, mismatch repair deficiency, and PD-L1 expression in a large patient cohort of gastrointestinal cancers. Mol. Cancer Res. 16(5), 805-812 (2018).

17. Chalmers ZR, Connelly CF, Fabrizio D et al. Analysis of 100,000 human cancer genomes reveals the landscape of tumor mutational burden. Genome Med. 9(1), 34 (2017).

18. Goodman AM, Kato S, Bazhenova L et al. Tumor mutational burden as an independent predictor of response to immunotherapy in diverse cancers. Mol. Cancer Ther. 16(11), 2598-2608 (2017).

19. Tian Q, Frierson HF Jr, Krystal GW, Moskaluk CA. Activating c-kit gene mutations in human germ cell tumors. Am. J. Pathol. 154(6), 1643-1647 (1999).

20. Cierna Z, Mego M, Miskovska V et al. Prognostic value of programmed-death-1 receptor (PD-1) and its ligand 1 (PD-L1) in testicular germ cell tumors. Ann. Oncol. 27(2), 300-305 (2016).

21. Chovanec M, Cierna Z, Miskovska V et al. Prognostic role of programmed-death ligand 1 (PD-L1) expressing tumor infiltrating lymphocytes in testicular germ cell tumors. Oncotarget 8(13), 21794-21805 (2017).

22. Adra N, Einhorn LH, Althouse SK et al. Phase II trial of pembrolizumab in patients with platinum refractory germ cell tumors: a Hoosier Cancer Research Network study GU14-206. Ann. Oncol. 29, 209-214 (2018).

23. Dieckmann KP, Radtke A, Spiekermann M et al. Serum levels of microRNA miR-371a-3p: a sensitive and specific new biomarker for germ cell tumours. Eur. Urol. 71(2), 213-220 (2017).

24. Mego M, Van Agthoven T, Gronesova P et al. Clinical utility of plasma miR-371a-3p in testicular germ cell tumors. J. Clin. Oncol. 36(Suppl.), e16540 (2018).

25. Albany C, Hever-Jardine MP, von Herrmann KM et al. Refractory testicular germ cell tumors are highly sensitive to the second generation DNA methylation inhibitor guadecitabine. Oncotarget 8(2), 2949-2959 (2017).

26. Taylor-Weiner A, Zack T, O’Donnell E et al. Genomic evolution and chemoresistance in germ-cell tumours. Nature 540(7631), $114-118$ (2016). 
